\title{
INVARIANT RATIOS AND FUNCTIONS IN GLASS DISPERSION
}

\author{
BY \\ P. G. NutTing
}

Designers of optical systems have for years been familiar with the fact that the ratios of any two partial disfusions is approximately the same constant for all optical glasses and have made use of the fact in selecting and ordering glasses.

Dr. F. E. Wright ${ }^{1}$ has recently made a careful study of these dispersion relations, plotting various partial dispersions against each other for practically all glasses (nearly 300 in all) for which data are available. His plotted curves are all straight lines upon which the data for all known glasses fall very closely. These straight lines do not however, quite pass through the origin nor through any other point in common. In other words, any partial dispersion such as $n_{\mathrm{F}}-n_{\mathrm{c}}$ is sensibly a linear function of any other partial dispersion such as $n_{\mathrm{D}}-n_{\mathrm{A}^{\prime}}$, but the ratio of the two is not quite invariant for all glasses:

Considerable interest has been aroused in this apparently significant and, at first sight, rather remarkable relation between refractive index and wave-length. The opinion appears to prevail that it indicates some.unique dispersion function to which the many heretofore advanced are mere approximations. A critical mathematical examination shows, however, that this deduction is not justified.

By way of introduction, consider first a simple linear function of $x$ and $y$ say

$$
y=a x+b
$$

in which $a$ and $b$ are variable parameters. This represents a double infinity of straight lines. Ordinates erected at any four fixed points on the $x$ axis, say $x_{1} x_{2} x_{3}$ and $x_{4}$, intersect all lines of both systems in the points $y_{1}=a x_{1}+b, y_{2}=$ etc. Now by eliminat-

${ }^{1}$ Jour. Opt. Soc. Am. 4, pp. 148-159, and 5, pp. 389-397, 1921. 
ing the variable parameters, an expression is obtained which is invariant for all lines of both systems, namely;

$$
\frac{y_{1}-y_{2}}{y_{3}-y_{4}}=\frac{x_{1}-x_{2}}{x_{3}-x_{4}}=\text { constant }
$$

Of the four points chosen, of course any two may be coincident. The second member of (2) would still be invariant and the elimination of $a$ and $b$ be unaffected if $x$ in (1) were given any exponent. However it is evident that the dependent variable $y$ must be of the first degree and must not occur with $x$ as a product. The most general form of function which will satisfy (2) appears to be that in which $y$ is any explicit linear function of any function of $x$ not involving parameters, or

$$
y=a f(x)+b
$$

in which $f(x)$ involves only numerical constants. This is certainly a sufficient and I believe a necessary condition for giving the invariant ratio of ordinate differences (2). It obviously covers a wide variety of functions and even classes of functions.

The application of these results to dispersion theory is simply a matter of translation. If refractive index $n$ is a function of wavelength $\lambda$ such that

$$
\frac{n_{1}-n_{2}}{n_{3}-n_{4}}=\text { constant }=c
$$

it is necessary and sufficient that the form of the dispersion function fall within the limitations

$$
n=a f(\lambda)+b
$$

in which, as in (3), $f(\lambda)$ involves only numerical constants while $a$ and $b$ vary from glass to glass. The limitations imposed by (4) and (5) of course fail to exclude many forms of dispersion functions which do not fit data at all.

Now Dr. Wright finds (l.c.) that (4) is not sufficiently general to fit known glass data but that

$$
n_{1}-n_{2}=c\left(n_{3}-n_{4}\right)+k
$$

holds very exactly for any fixed set of four wave-lengths. The added constant $k$, although small (.0002 to .0008 and either positive or negative), is a very definite quantity and by no means to be regarded as merely a second order term giving a closer 
approximation. The reason for this finite value of $k$ is not apparent for the Cauchy and other dispersion formulas known to fit glass dispersion data quite well fall within class (5) and satisfy (4) and therefore imply $k=0$.

The limitations set by (6) are broader even than those set by (4) unless perchance a direct functional relation exists between $c$ and $k$. The most general relation between index and wavelength found by the writer to satisfy (6) is

$$
n=a f(\lambda)+b+F(\lambda)
$$

and this is believed to be both sufficient and necessary for the existence of (6). In (7) both $f(\lambda)$ and $F(\lambda)$ are unlimited except each must involve only numerical constants. Any function which will satisfy (6) will of course satisfy (4), but the converse is not true.

Hence while such a simple relation as (4) or the more exact relation (6) may be useful in such practical work as mixing glass batches and selecting glasses for lens systems, they cannot be regarded as indicating any specific dispersion formula or even as setting any but very wide limitations on such functions in general.

DEC. 11, 1921. 